\title{
Post-Stroke Blood-Brain Barrier Disruption and Poor Functional Outcome in Patients Receiving Thrombolytic Therapy
}

\author{
Zurab Nadareishvili Alexis N. Simpkins Emi Hitomi Dennys Reyes \\ Richard Leigh \\ National Institute of Neurological Disorders and Stroke, National Institutes of Health, Bethesda, MD, USA
}

\section{Keywords}

Blood-brain barrier · Functional outcome $\cdot$ Ischemic stroke . Magnetic resonance imaging · Post-stroke inflammation

\begin{abstract}
Background and Purpose: The role played by post-stroke inflammation after an ischemic event in limiting functional recovery remains unclear. One component of post-stroke inflammation is disruption of the blood-brain barrier (BBB). This study examines the relationship between post-stroke BBB disruption and functional outcome. Methods: Acute stroke patients treated with thrombolysis underwent magnetic resonance imaging scanning $24 \mathrm{~h}$ and 5 days after their initial event. BBB permeability maps were generated from perfusion weighted imaging. Average permeability was calculated in the affected hemisphere. Good functional outcome, defined as a modified Rankin score of 0 or 1 , was compared with average permeability using logistic regression. Results: Of the 131 patients enrolled, 76 patients had the necessary data to perform the analysis at $24 \mathrm{~h}$, and 58 patients had data for the 5-day assessment. Higher BBB permeability measured at $24 \mathrm{~h}(\mathrm{OR} 0.57 ; 95 \% \mathrm{Cl} 0.33-0.99, p=0.045)$ and at 5 days (OR $0.24 ; 95 \% \mathrm{Cl} 0.09-0.66, p=0.005$ ) was associ-
\end{abstract}

ated with worse functional outcome 1-3 months after the acute ischemic stroke. For every percentage increase in BBB disruption at 5 days, there was a $76 \%$ decrease in the chance of achieving a good functional outcome after stroke. Multivariate analysis found this to be independent of age, stroke volume, or clinical stroke severity. Conclusions: Post-stroke BBB disruption appears to be predictive of functional outcome irrespective of stroke size.

ㄷ 2019 S. Karger AG, Basel

\section{Introduction}

Acute ischemic stroke (AIS) causes immediate brain injury due to a lack of sufficient blood flow to the affected brain tissue. Treatments for AIS aim to restore blood flow either mechanically with thrombectomy or pharmacologically with thrombolysis using intravenous (IV) tissue plasminogen activator (tPA). Successful early reperfusion is associated with better functional outcomes. In the days after an AIS, a post-stroke inflammatory response occurs [1]; however, the role of this post-stroke inflammation in functional outcome is yet to be established.

\section{KARGER}

(c) 2019 S. Karger AG, Basel

E-Mail karger@karger.com

www.karger.com/ced 
In a healthy brain, the blood-brain barrier (BBB) regulates the entry of cells and molecules into the brain which, in conjunction with the central nervous system (CNS) microenvironment, creates a certain degree of immune privilege [2]. The BBB is known to become disrupted during and after an AIS. There is an early, reversible opening of the $\mathrm{BBB}$ during the hours after stroke, which is followed by a second irreversible disruption of the $\mathrm{BBB}$ in the days after the stroke due to an inflammatory response [1]. Clinically, this neuroinflammatory phase is seen as gadolinium enhancement on magnetic resonance imaging (MRI) when performed several days after the AIS [3].

In this study, we quantified BBB disruption on MRI as a biomarker for post-stroke neuroinflammation during the days after thrombolysis for AIS in order to investigate the role of this measure in functional outcome.

\section{Methods}

\section{Population}

All patients included in this study were enrolled in our Institutional Review Board (IRB)-approved National Institutes of Health (NIH) Natural History of Stroke study (identification number NCT00009243), which is an observational cohort study of stroke patients. Patients enrolled in the Natural History of Stroke study, whenever possible, have MRIs performed $24 \mathrm{~h}$ after treatment and again approximately 5 days after their stroke. Patients enrolled during 2013 and 2014, who had an MRI scan prior to treatment with IV tPA and follow-up MRI imaging with gadolinium at the $24 \mathrm{~h}$ and/or the 5-day time points after their AIS, were included in this study if they had been affected by a unilateral supratentorial stroke. This study required comparison between the affected and unaffected hemispheres to perform the BBB analysis; thus, patients with bilateral strokes were excluded.

\section{MRI Parameters}

Images were acquired on a 1.5T GE Signa scanner (General Electric Medical Systems, Milwaukee, WI, USA), a 3T Philips Achieva scanner (Philips Healthcare, Best, The Netherlands), or a 3T Siemens Skyra scanner (Siemens AG, Munich, Germany). Image sequences and typical parameter ranges were as follows: Diffusion tensor imaging (TR 4,461-10,500 ms, TE 61.6-91.3 ms, $3.5 \mathrm{~mm}$ slice thickness, 40 slices) used to generate trace diffusion weighted images using 3 orthogonal directions $(b=0$ and $1,000 \mathrm{~s} / \mathrm{mm}^{2}$ ) and apparent diffusion coefficient (ADC) maps; dynamic susceptibility contrast perfusion weighted imaging (PWI; TR 1-1.5 s, TE 25-45 ms, 7 mm slice thickness, 20 slices, 40-80 dynamics), which was collected during the injection of a weight-based dose of gadolinium; FLAIR imaging (TR 9,000 ms, TE 120-145 ms, $3.5 \mathrm{~mm}$ slice thickness, 40 slices); time-of-flight magnetic resonance angiography images (TR 18-23 ms, TE 3.43-6.8 ms, 0.75-1.4 mm slice thickness, 73-95 slices); and gradient recall echo images (TR 700-800 ms, TE $12-20.4 \mathrm{~ms}$, 3.5-7 mm slice thickness, $20-40$ slices).

\section{Image Processing}

MRI scans were co-registered to a template atlas using a diffeomorphic registration algorithm [4]. The template atlas was used to segment the left and right hemispheres and remove infratentorial structures. BBB permeability maps were generated from the PWI source images using a method previously described [5-7]. BBB permeability of the affected hemisphere was calculated relative to the unaffected hemisphere on a voxel-by-voxel basis, expressed as a percent increase over normal. Voxels in the affected hemisphere that demonstrated increased BBB permeability above the noise threshold of $1 \%$ were used to generate the average permeability of the hemisphere. The size of the pre-treatment perfusion deficit was calculated from the PWI using a time-to-peak threshold of $4 \mathrm{sec}$ onds beyond normal tissue. Time-to-peak threshold was used instead of time-to-maximum, since it has been shown to be reasonably interchangeable with time-to-maximum; it is more easily reproduced, and it is less susceptible to artifacts introduced by deconvolution $[8,9]$. The final infarct volume was calculated from the 24-h MRI scan as per recommended guidelines [10] using an ADC threshold of $600 \mu \mathrm{m}^{2} / \mathrm{s}$. The 24 -h time point was used to calculate infarct volume to avoid the effects of vasogenic edema and normalization of ADC values that can occur at the 5-day time point [10]. Image processing was performed in Matlab (Mathworks, Natick, MA, USA). Image processing was done using an automated pipeline with minimal user interaction. The BBB calculations were completely automated and thus not influenced by an operator or other variables as described previously [11].

\section{Clinical and Outcome Measures}

Stroke severity was determined at the 5-day time point based on the NIH Stroke Scale (NIHSS). If a patient was discharged prior to the 5-day time point, the NIHSS at the time of discharge was used as the 5-day value. Hemorrhagic transformation complications were assessed on the MRI at the 24-h time point. New hemorrhage since the pre-thrombolysis MRI was graded according to ECASS criteria [12]. Patients were assessed for any hemorrhage, parenchymal hematoma, or symptomatic intracranial hemorrhage (sICH) based on SITS-MOST criteria [13]. Large vessel occlusion was defined as occlusion of the internal carotid artery or the first segment of the middle cerebral artery on the pretreatment magnetic resonance angiography. Chronic cerebral small vessel disease was assessed by applying the Fazekas scale [14] to the pretreatment FLAIR scan. Functional outcome was assessed based on the modified Rankin scale (mRS), evaluated by stroke researchers certified in performing this evaluation. Patients who returned for an MRI 1 month after their acute event had their 1-month mRS assessed during that visit. Patients who could be contacted by phone 3 months after their event had their 3-month mRS calculated based on that phone interview. Final mRS used for this study was based on the 3-month mRS if that data point was available. In the absence of a 3-month mRS, the 1-month mRS was used as the final mRS. Patients without either a 1-month or a 3-month mRS were excluded from this study. Good functional outcome was defined as a final mRS of 0 or 1 , since this is the standard definition used in studies of thrombolysis [15]. Outcome assessments were conducted blinded to the results of the BBB analysis.

\section{Statistical Analysis}

Average permeability was treated as a continuous independent variable and compared with the continuous dependent variable 
$\mathrm{mRS}$ using linear regression. Average permeability was also treated as a continuous independent variable when compared with the binary dependent variable good functional outcome using logistic regression. A $p$ value $<0.05$ was considered significant. Receiver operator characteristic analysis was used to compare the 24 -h and 5 -day average $\mathrm{BBB}$ values to determine the value that was better at predicting a good functional outcome. To compare the degree of $\mathrm{BBB}$ disruption at $24 \mathrm{~h}$ with the degree at 5 days in patients with a good functional outcome, a mean comparison $t$ test was used. Demographics, vascular risk factors, stroke severity, perfusion deficit, and final infarct volume where included in a logistic regression multivariate analysis comparing average permeability with good functional outcome if they had a $p$ value $<0.1$ in univariate analysis. All statistical analyses were performed using STATA 13 (StataCorp LLC, College Station, TX, USA).

\section{Availability of Data and Materials}

Data in this study were monitored by the NIH Office of Human Subjects Research Protections and the Combined NeuroScience (CNS) IRB of NIH. Requests for access to the data may be possible if approved by these governing bodies. Please contact the corresponding author for data requests.

\section{Results}

Of the 131 tPA-treated patients who were evaluated with MRI and enrolled during the study period, 76 patients met the inclusion criteria for this sub-study and had the necessary imaging at the 24 -h time point. For the 5 -day time point, 58 patients had the necessary imaging. These 2 cohorts overlapped by 50 patients who had both timepoints. The breakdown for the population is shown diagrammatically in Figure 1. Average permeability measured at $24 \mathrm{~h}\left(p=0.012, r^{2}=0.08\right)$ and 5 days $(p<0.001$, $\left.r^{2}=0.19\right)$ was significantly associated with mRS $1-3$ months later. However, the $r^{2}$ terms suggest that the role of $\mathrm{BBB}$ disruption in determining outcome is twice as important at 5 days than at $24 \mathrm{~h}$.

Looking at the binary outcome of good functional outcome ( $\mathrm{mRS}$ of 0 or 1 ), average permeability was significant when measured at $24 \mathrm{~h}(p=0.045$, OR $0.57 ; 95 \% \mathrm{CI}$ $0.33-0.99$ ) but even more so at 5 days ( $p=0.005$, OR 0.24; $95 \%$ CI $0.09-0.66)$. This indicates that for every $1 \%$ increase in the BBB permeability measured 5 days after stroke, the chance of achieving a good functional outcome decreases by $75 \%$. A boxplot showing the difference in day 5 BBB disruption between the outcome groups is shown in Figure 2 .

Receiver operator characteristic analysis testing the predictive value of average permeability in determining if a patient will have a good outcome found an area under the curve of 0.67 for the $24-\mathrm{h} \mathrm{BBB}$ and an area under the curve of 0.77 for the 5 -day BBB. This confirms that 5-day
$\mathrm{BBB}$ disruption plays a more substantial role in determining functional outcome than the BBB measured at 24-h. Looking only at patients who had a good functional outcome, average BBB was significantly higher at $24 \mathrm{~h}$ than at 5 days $(p=0.04)$ as seen in Figure 3. This suggests that some portion of the BBB disruption detected at the earlier time point was reversible in patients who did well, again supporting the notion that 5 -day $\mathrm{BBB}$ disruption is a better predictor of outcome.

Given the stronger association with 5 -day $\mathrm{BBB}$, the subsequent analysis focused only on the 5-day BBB measurements. Table 1 shows the characteristics of the 58 patients included in the 5-day BBB analysis separated by those who did, and did not, achieve a good functional outcome. Overall, this cohort had predominantly mild strokes with a median NIHSS of 3 at the time of the 5-day scan and fairly good outcomes with a median mRS of 2 . Large vessel occlusion was present in 14 patients, 7 had a good functional outcome, and 7 did not. At the time of the study, the use of endovascular treatment had not been validated; however, 3 patients in this cohort did have endovascular treatment in addition to IV thrombolysis, and all had a good functional outcome.

Although strokes were on average larger in the poor outcome group, this did not reach significance. Good functional outcome was associated with younger age $(p=0.003)$ and lower NIHSS at 5 days $(p=0.043)$. Chronic small vessel disease trended toward a larger white matter lesion load in patients with a poor outcome $(p=0.060)$. In multivariate analysis, 5-day average permeability remained independent of age ( $p=0.016$, OR $0.33 ; 95 \%$ CI $0.13-0.82)$, NIHSS at 5 days ( $p=0.019$, OR $0.27 ; 95 \%$ CI $0.09-0.80)$ or Fazekas score $(p=0.008$, OR $0.26 ; 95 \%$ CI $0.10-0.71)$. None of the variables included in the multivariate analysis was identified as being collinear with 5-day average permeability.

\section{Discussion}

This study used a quantitative measure of gadolinium leakage through the $\mathrm{BBB}$ as a component measure of post-stroke inflammation. We identified a range of values across the population suggesting that post-stroke inflammation is variable between patients in a manner that is independent of stroke size or severity. Patients with more post-stroke inflammation 5 days after their ischemic event were more likely to have a poor outcome 1-3 months later. In our cohort, BBB permeability had a stronger relationship with outcome than the final infarct volume. Final infarct volume reflects the injury that has 
Fig. 1. A flowchart shows how the original population of patients enrolled in the study were either included or excluded to arrive at the final cohort populations. Note the 50 patients who had both time points included in both cohorts. NIH, National Institutes of Health.

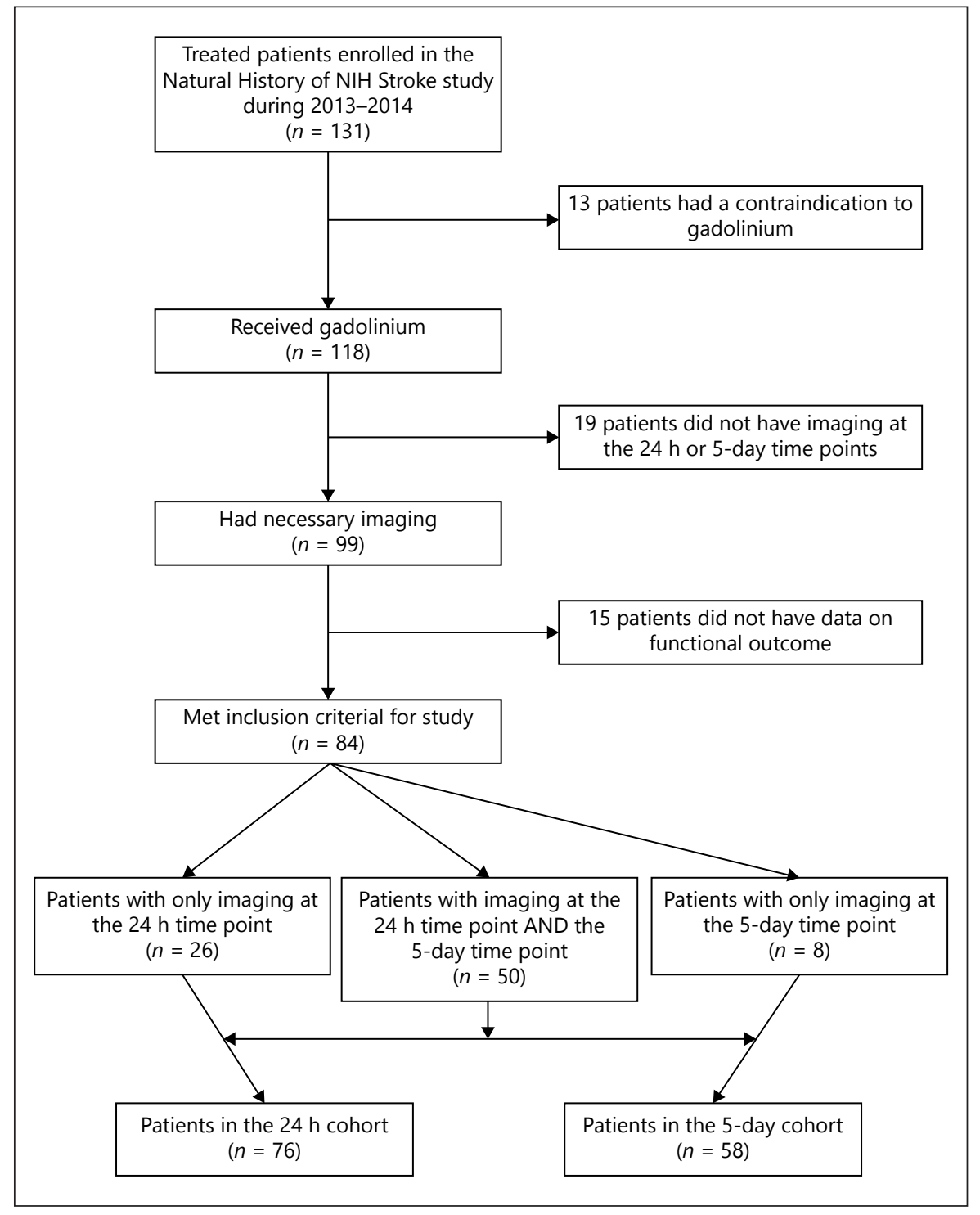

already occurred, while BBB disruption presumably reflects ongoing injury and thus may be a better predictor of functional outcome over the subsequent months.

The use of BBB disruption as a marker for post-stroke inflammation performed better at the 5-day time point than the 24-h time point, which is consistent with the known time course of BBB disruption and its relationship to post-stroke inflammation. BBB permeability has been shown to be dynamic in animal models of stroke [16] and also in humans [7]. BBB disruption early after stroke has been attributed to hypoxia-induced activation of latent matrix metalloproteinase 2 and is reversible [1]. However, a second phase of $\mathrm{BBB}$ disruption can occur in the days after a stroke mediated by MMP-3, MMP-9, and cyclooxygenases 2 [17]. This inflammatory opening of the BBB leads to neutrophil infiltration, cerebral edema, microglial activation, and the production of neuroinflammatory mediators that exacerbate the cerebral injury [16].

Cerebral ischemia-related inflammation engages both the innate and adaptive immune systems with innate immunity being activated in the first $24-48 \mathrm{~h}$, and adaptive responses occurring several days later [18]. In animal models of ischemia-reperfusion, peak inflammation was observed 3-7 days after the ischemic event [19]. This inflammation peak was associated with a high infiltration rate of not only neutrophils but also adaptive immune system cells, which were represented by dendritic cells, T-cells, and NK cells [19].

This peak of inflammation occurs during the second phase of BBB, which allows the invasion of immune cells 


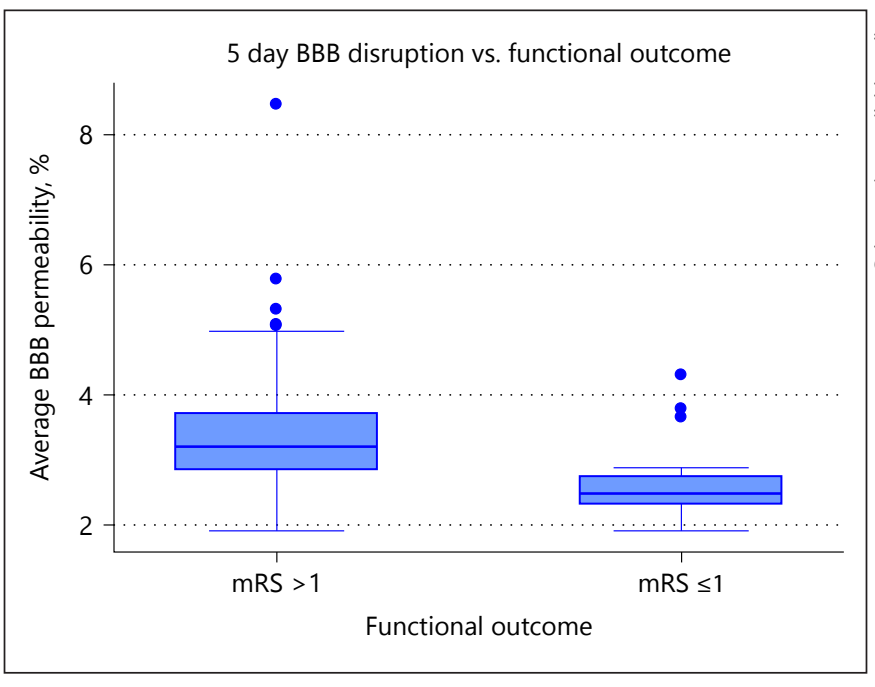

Fig. 2. A box plot compares the BBB measured on day 5 between patients who had a good outcome $(m R S \leq 1)$ with patients who did not (mRS $>1$ ). BBB, blood-brain barrier; mRS, modified Rankin scale.

into the infarcted brain. These cells, upon encountering novel brain antigens, initiate an autoimmune response, which leads to further destruction of neurological tissue [20]. Pathological studies in humans have shown that an inflammatory infiltrate persists after the stroke between days 3 and 37 and is characterized by the presence of polymorphonuclear leucocytes and mononuclear cells in $84 \%$ and $41 \%$ of cases respectively [21]. Given the existing evidence that the second phase of $\mathrm{BBB}$ disruption on days 3-7 coincides with the peak of proinflammatory cell infiltration in infarcted tissue, it is likely that inflammatory immune reactions, characterized by $\mathrm{BBB}$ disruption, are responsible for the unfavorable stroke outcome seen in our study.

If proinflammatory immune response and BBB disruption are associated with unfavorable stroke outcome, could anti-inflammatory interventions improve stroke outcome? Thus far, there is limited clinical evidence that anti-inflammatory therapy improves stroke outcome. A phase II, randomized, double-blind, placebo-controlled clinical trial of recombinant human IL-1ra in patients with acute stroke showed that, among patients with cortical infarcts, clinical outcomes at 3 months in the rhIL-1ratreated group were better than those in the placebo group [22]. Minocycline, the antibiotic with anti-inflammatory and protease inhibitor properties, has been shown to be safe when administered together with IV t-PA [23]. In another open-label study, minocycline appeared to improve NIHSS, mRS, and Barthel Index [24]. However, it is also possible that the $\mathrm{BBB}$ disruption itself is facilitating the

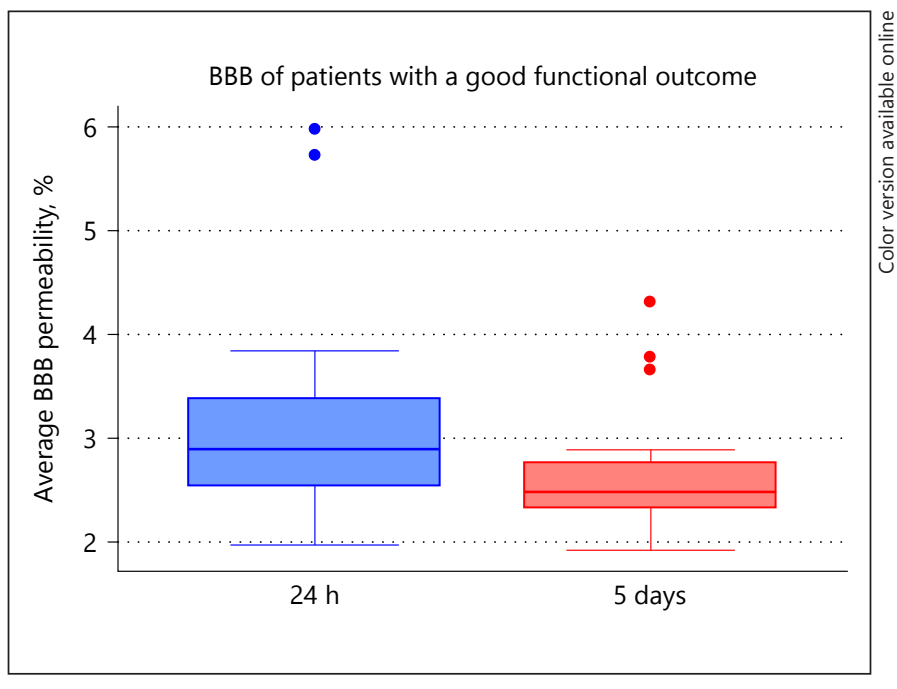

Fig. 3. A box plot compares the BBB measured $24 \mathrm{~h}$ after stroke with the $\mathrm{BBB}$ measured 5 days after stroke for patients who had a good outcome $(\mathrm{mRS} \leq 1)$. BBB, blood-brain barrier.

ongoing injury leading to poor outcome. Thus, treatments targeting BBB stabilization may also play a role. One approach is to identify agents that inhibit MMP activity [25].

Natalizumab, which is anti-a4-integrin monoclonal antibody, was tested in a phase II, randomized, placebocontrolled clinical trial in AIS [26] and was found to be associated with better functional outcome in the treatment group compared to placebo. Natalizumab is known, in multiple sclerosis, to prevent white blood cells from crossing the $\mathrm{BBB}$ and attacking the CNS [27]. It is possible that favorable clinical outcome in the stroke trial was achieved through the modulation of late proinflammatory effects associated with the prevention of T-cell traffic through disrupted BBB. A follow-up study of natalizum$a b$ in stroke was reportedly negative. However, the measurement of BBB disruption prior to enrollment has not been part of any clinical trial testing anti-inflammatory medications in stroke patients. The present study indicates that the measurement of BBB leakage may provide a useful biomarker for future trials of anti-inflammatory agents as promoters of recovery from stroke.

MRI has previously been used to measure post-stroke gadolinium leakage in stroke patients [28]. This previous study also found variable levels of BBB disruption across the population that appeared to peak in the timeframe of 6-48 $\mathrm{h}$ after stroke; however, that study had neither the serial imaging nor the temporal resolution to identify the fluctuations seen in the current study. Our study used a method for measuring BBB disruption that involved post 
Table 1. The population of patients who had BBB measured 5 days after stroke is characterized and separated by outcome

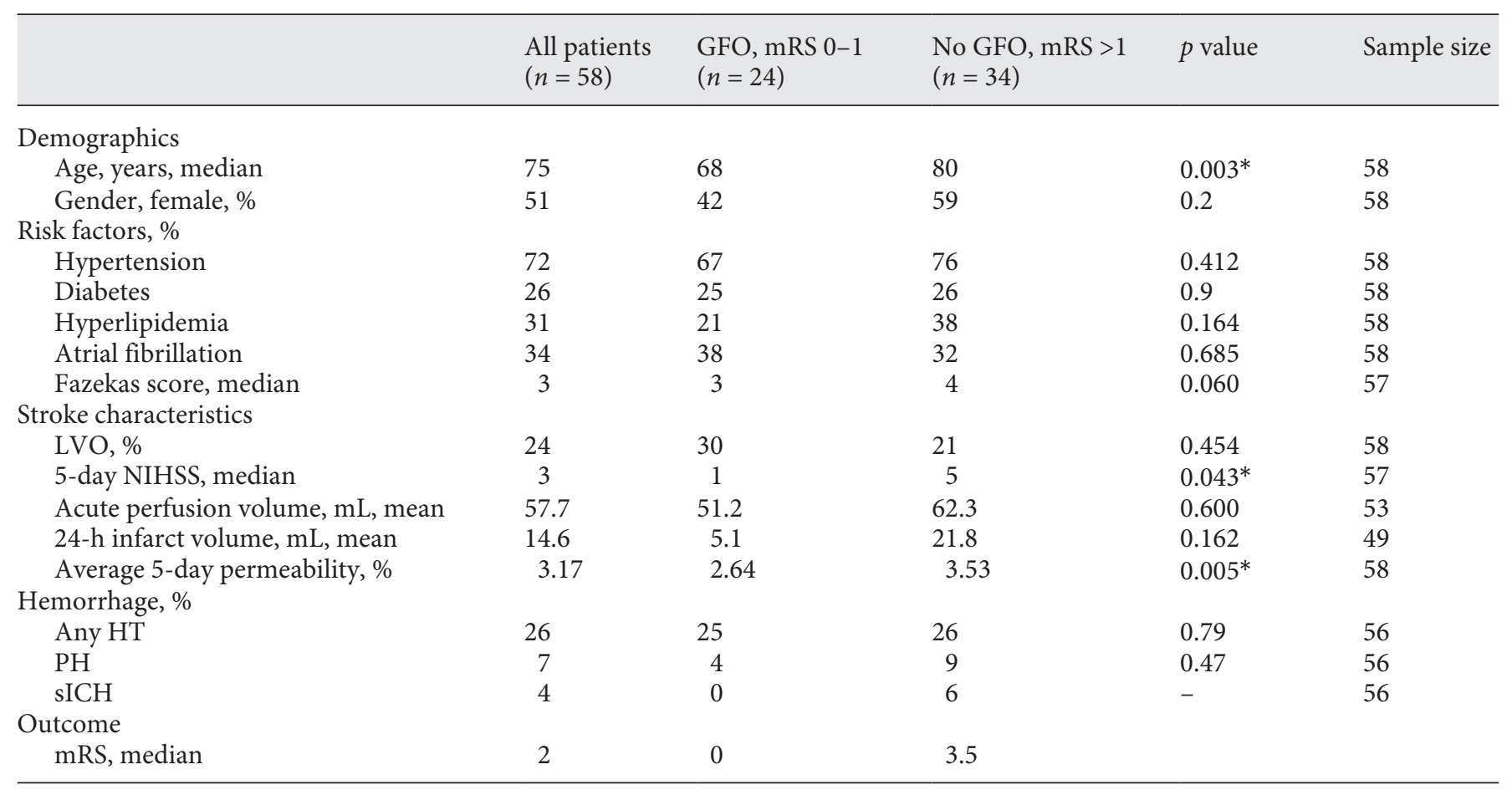

* Significant difference.

The $p$ values reflect the significance of differences between the groups based on the listed characteristic.

Sample size indicates the number of patients who had the necessary information to be part of the logistic regression.

BBB, blood-brain barrier; GFO, good functional outcome; LVO, large vessel occlusion; NIHSS, National Institutes of Health Stroke Scale; HT, hemorrhagic transformation; PH, parenchymal hematoma; sICH, symptomatic intracranial hemorrhage; mRS, modified Rankin Scale.

processing of an MRI sequence that is routinely acquired as part of a clinical stroke evaluation $[5,29,30]$. Thus, in identifying a link between post-stroke BBB disruption and functional outcome, a biomarker for identification of patients who may benefit from a therapeutic intervention may also have been identified.

We previously reported increased BBB permeability in patients with chronic small vessel disease [11]. This form of $\mathrm{BBB}$ disruption is also thought to be inflammatory in nature [1]. In the current study, we controlled for chronic white matter hyperintensities and found that elevated BBB permeability at 5 days was associated with poor outcome independent of the baseline burden of white matter lesions. However, there may be a link between acute and chronic BBB disruption in cerebrovascular disease, and further studies are needed. Increased BBB permeability is a known risk factor for sICH [5], and idiopathic ICH itself causes opening of the BBB. sICH was rare in our cohort and occurred only in the poor outcome group; thus, we are not able to separate the role of sICH versus the 5 -day
$\mathrm{BBB}$ measurement in functional outcome. However, removing the 2 patients with sICH from the logistic regression did not impact the relationship between 5-day BBB and functional outcome $(p=0.006)$.

There are several limitations to this study; the sample size was modest and varied based on the available data. However, this did not lead to marginal significance or a small effect size with regard to the primary hypothesis. There may be selection bias, since only patients who signed informed consent to be part of our observational study were included, and among those who participated, only those with adequate data were included in the analysis. Because of this, it is also possible that patients with more severe strokes were not included in the study. Since patients with poor renal function are unable to received gadolinium, they were excluded from this study. As such, these results may not generalize to them. All patients in this study received IV tPA, which may have affected the BBB; thus, it is not known if these results would apply to an untreated population. Also, despite the significant circumstantial support pre- 
sented above, we do not know definitively that the BBB measured is a true reflection of the inflammatory response. Similarly, we do not know that treatment of the measured BBB disruption would change the outcome. Future studies should test the effect of anti-inflammatory drugs on poststroke BBB disruption to further characterize these points.

\section{Conclusions}

This study found that higher degrees of BBB disruption, measured on MRI 5 days after thrombolytic therapy for acute stroke, were associated with worse outcome despite prior treatment with acute reperfusion therapy and independent of stroke size or severity. Thus, BBB leakage as a biomarker for post-stroke inflammation may constitute a useful therapeutic index for treatment in future studies of ischemic stroke.

\section{Acknowledgments}

This research was possible because of contributions from the NIH Natural History of Stroke Investigators including R.T.B., A.W.H., L.L.L., R.L., M.L., J.K.L., and Z.N.

\section{Statement of Ethics}

This study was approved by the CNS IRB of the NIH. All patients, or their legally authorized representative, signed informed consent.

\section{Disclosure Statement}

The authors declare that they have no conflicts of interest to disclose.

\section{Funding Sources}

Funding for this study came from the National Institute of Neurological Disorders and Stroke Intramural Research Program of the NIH.

\section{Authors Contribution}

Z.N. played a major role in acquisition of the data and in drafting the manuscript. A.N.S. and D.R. revised the manuscript for intellectual content. E.H. analyzed the data. R.L. designed and conceptualized the study, analyzed the data, and drafted the manuscript.

\section{References}

1 Yang Y, Rosenberg GA. Blood-brain barrier breakdown in acute and chronic cerebrovascular disease. Stroke. 2011 Nov;42(11):33238.

2 Galea I, Bechmann I, Perry VH. What is immune privilege (not)? Trends Immunol. 2007 Jan;28(1):12-8.

3 Karonen JO, Partanen PL, Vanninen RL, Vainio PA, Aronen HJ. Evolution of MR contrast enhancement patterns during the first week after acute ischemic stroke. AJNR Am J Neuroradiol. 2001 Jan;22(1):103-11.

4 Oishi K, Faria A, Jiang H, Li X, Akhter K, Zhang J, et al. Atlas-based whole brain white matter analysis using large deformation diffeomorphic metric mapping: application to normal elderly and Alzheimer's disease participants. Neuroimage. 2009 Jun;46(2):486-99.

5 Leigh R, Jen SS, Hillis AE, Krakauer JW, Barker PB, Albers GW, et al.; STIR and VISTA Imaging Investigators. Pretreatment bloodbrain barrier damage and post-treatment intracranial hemorrhage in patients receiving intravenous tissue-type plasminogen activator. Stroke. 2014 Jul;45(7):2030-5.

6 Leigh R, Christensen S, Campbell BC, Marks MP, Albers GW, Lansberg MG; DEFUSE 2 Investigators. Pretreatment blood-brain barrier disruption and post-endovascular intra- cranial hemorrhage. Neurology. 2016 Jul; 87(3):263-9.

7 Simpkins AN, Dias C, Leigh R, Benson RT, Hsia AW, Latour LL, et al.; National Institutes of Health Natural History of Stroke Investigators. National Institutes of Health Natural History of Stroke I. Identification of reversible disruption of the human blood-brain barrier following acute ischemia. Stroke. 2016 Sep;47(9):2405-8.

8 Zaro-Weber O, Moeller-Hartmann W, Heiss WD, Sobesky J. Maps of time to maximum and time to peak for mismatch definition in clinical stroke studies validated with positron emission tomography. Stroke. 2010 Dec; 41(12):2817-21.

9 Wouters A, Christensen S, Straka M, Mlynash $\mathrm{M}$, Liggins J, Bammer R, et al. A comparison of relative time to peak and Tmax for mismatch-based patient selection. Front Neurol. 2017 Oct; $8: 539$.

10 Warach SJ, Luby M, Albers GW, Bammer R, Bivard A, Campbell BC, et al.; Stroke Imaging Research (STIR) and VISTA-Imaging Investigators. Acute stroke imaging research roadmap iii imaging selection and outcomes in acute stroke reperfusion clinical trials: consensus recommendations and further research priorities. Stroke. 2016 May;47(5): 1389-98.
11 Arba F, Leigh R, Inzitari D, Warach SJ, Luby M, Lees KR; STIR/VISTA Imaging Collaboration. Blood-brain barrier leakage increases with small vessel disease in acute ischemic stroke. Neurology. 2017 Nov;89(21):214350.

12 Hacke W, Kaste M, Fieschi C, von KR, Davalos A, Meier D, et al. Randomised double-blind placebo-controlled trial of thrombolytic therapy with intravenous alteplase in acute ischaemic stroke (ECASS II). Second european-australasian acute stroke study investigators. Lancet. 1998;352:1245-51.

13 Wahlgren N, Ahmed N, Dávalos A, Ford GA, Grond M, Hacke W, et al.; SITS-MOST investigators. Thrombolysis with alteplase for acute ischaemic stroke in the Safe Implementation of Thrombolysis in Stroke-Monitoring Study (SITS-MOST): an observational study. Lancet. 2007 Jan;369(9558):275-82.

14 Fazekas F, Chawluk JB, Alavi A, Hurtig HI Zimmerman RA. MR signal abnormalities at 1.5 T in Alzheimer's dementia and normal aging. AJR Am J Roentgenol. 1987 Aug;149(2): 351-6.

15 National Institute of Neurological Disorders and Stroke rt-PA Stroke Study Group. Tissue plasminogen activator for acute ischemic stroke. N Engl J Med. 1995;333(24):1581-7. 
16 Sandoval KE, Witt KA. Blood-brain barrier tight junction permeability and ischemic stroke. Neurobiol Dis. 2008 Nov;32(2):20019.

17 Candelario-Jalil E, González-Falcón A, García-Cabrera M, León OS, Fiebich BL. Postischaemic treatment with the cyclooxygenase-2 inhibitor nimesulide reduces bloodbrain barrier disruption and leukocyte infiltration following transient focal cerebral ischaemia in rats. J Neurochem. 2007 Feb; 100(4):1108-20.

18 Iadecola C, Anrather J. The immunology of stroke: from mechanisms to translation. Nat Med. 2011 Jul;17(7):796-808.

19 Gelderblom M, Leypoldt F, Steinbach K, Behrens D, Choe CU, Siler DA, et al. Temporal and spatial dynamics of cerebral immune cell accumulation in stroke. Stroke. 2009 May; 40(5): 1849-57.

20 Meisel C, Schwab JM, Prass K, Meisel A, Dirnagl U. Central nervous system injury-induced immune deficiency syndrome. Nat Rev Neurosci. 2005 Oct;6(10):775-86.
21 Mena H, Cadavid D, Rushing EJ. Human cerebral infarct: a proposed histopathologic classification based on 137 cases. Acta Neuropathol. 2004 Dec;108(6):524-30.

22 Emsley HC, Smith CJ, Georgiou RF, Vail A, Hopkins SJ, Rothwell NJ, et al.; Acute Stroke Investigators. A randomised phase II study of interleukin-1 receptor antagonist in acute stroke patients. J Neurol Neurosurg Psychiatry. 2005 Oct;76(10):136672.

23 Fagan SC, Waller JL, Nichols FT, Edwards DJ, Pettigrew LC, Clark WM, et al. Minocycline to improve neurologic outcome in stroke (MINOS): a dose-finding study. Stroke. 2010 Oct;41(10):2283-7.

24 Lampl Y, Boaz M, Gilad R, Lorberboym M, Dabby R, Rapoport A, et al. Minocycline treatment in acute stroke: an open-label, evaluator-blinded study. Neurology. 2007 Oct; 69(14):1404-10.

25 Yang Y, Rosenberg GA. Matrix metalloproteinases as therapeutic targets for stroke. Brain Res. 2015 Oct;1623:30-8.
26 Elkins J, Veltkamp R, Montaner J, Johnston SC, Singhal AB, Becker K, et al. Safety and efficacy of natalizumab in patients with acute ischaemic stroke (ACTION): a randomised, placebo-controlled, double-blind phase 2 trial. Lancet Neurol. 2017 Mar;16(3):217-26.

27 Bauer M, Brakebusch C, Coisne C, Sixt M, Wekerle H, Engelhardt B, et al. Betal integrins differentially control extravasation of inflammatory cell subsets into the CNS during autoimmunity. Proc Natl Acad Sci USA. 2009 Feb;106(6):1920-5.

28 Merali Z, Huang K, Mikulis D, Silver F, Kassner A. Evolution of blood-brain-barrier permeability after acute ischemic stroke. PLoS One. 2017 Feb;12(2):e0171558.

29 Leigh R, Jen SS, Varma DD, Hillis AE, Barker PB. Arrival time correction for dynamic susceptibility contrast MR permeability imaging instrokepatients.PLoSOne.2012;7(12):e52656.

30 Leigh R, Krakauer JW. MRI-guided selection of patients for treatment of acute ischemic stroke. Curr Opin Neurol. 2014 Aug;27(4): 425-33. 